\title{
Intractable Methicillin-Resistant Staphylococcus Aureus Otorrhea with Silicone Impression in the Middle Ear
}

\author{
Eun Kyung Jung, Chul Ho Jang, Jong Yuap Seong, and Yong Beom Cho \\ Department of Otolaryngology, Chonnam National University Medical School, Gwangju, Korea
}

\author{
Received July 14, 2016 \\ Revised September 14, 2016 \\ Accepted September 26, 2016

\section{Address for correspondence Chul Ho Jang, MD \\ Department of Otolaryngology, \\ Chonnam National University \\ Medical School, 42 Jebong-ro, \\ Dong-gu, Gwangju 61469, Korea \\ Tel $+82-62-220-6774$ \\ Fax +82-62-220-6776 \\ E-mail chulsavio@hanmail.net}

We report a case of methicillin-resistant Staphylococcus aureus (MRSA) otorrhea with impression material of hearing aid in the middle ear. The patient had chronic otitis media in the right ear with sensorineural hearing loss in both ears. The silicone flowed into the middle ear through a tympanic membrane perforation during the process of making an ear mold. Several days after hearing aid fitting, the patient had severe otalgia, intractable otorrhea, aggravated hearing loss, and dizziness. The pus culture and sensitivity test revealed MRSA. After topical treatment using diluted vinegar irrigation and ototopical vancomycin solution, intractable otorrhea was controlled. The infected silicone impression was removed by canal wall-up mastoidectomy, and hearing was saved. We present here a review of the literature regarding silicone impression in the middle ear after hearing aid mold fitting.

J Audiol Otol 2017;21(1):49-52

KEY WORDS: Silicone impression · Hearing aid · MRSA · Otorrhea $\cdot$ Middle ear.

\section{Introduction}

Complications during aural impression-taking for hearing aids are relatively rare. There are several reports in the literature of complications related to ear mold impression material [1-6]. Most cases occurred when there was preexisting perforation of the tympanic membrane, although traumatic perforation occurred in rare cases. While impacted mold material in the open mastoid cavity or external ear canal can be easily removed without complications, removal of the foreign body from the middle ear is challenging and should not be undertaken by inexperienced operators.

To date, transcanal or meatoplasty are the most commonly used approaches. However, a dumbbell-shaped mass impacted in the middle ear cavity cannot be removed by transcanal approach. Furthermore, while symptoms and surgical treatment for impression material in the middle ear have been reported [1-8], a case of impression material in the middle ear with accompanying methicillin-resistant Staphylococcus au-

This is an Open Access article distributed under the terms of the Creative Commons Attribution Non-Commercial License (http://creativecommons.org/licenses/by-nc/4.0/) which permits unrestricted non-commercial use, distribution, and reproduction in any medium, provided the original work is properly cited. reus (MRSA) otorrhea has not. We have experienced a case of silicone impression material in the middle ear with MRSA otorrhea.

We report the findings for a 74-year-old man who suffered intractable pulsating purulent discharge with otalgia following fitting of the silicone mold for a hearing aid. A large amount of silicone impression material thus impacted the middle ear like an iceberg. After topical treatment of the MRSA otorrhea by vancomycin otic solution and diluted vinegar solution irrigation, we removed the impression material using canal wallup mastoidectomy. We present here our review of the literature regarding such a case.

\section{Case Report}

Several days after being diagnosed with simple chronic otitis media without otorrhea at the local clinic, he visited a local shop selling hearing aids. After hearing aid molding was performed, the mold could not be removed. Ten days after hearing aid fitting, patient had severe otalgia, aggravated hearing loss, and dizziness. Otomicroscopic examination found pulsating purulent discharge with blue mold material from the middle ear (Fig. 1A). The pulsating discharge was 
collected using a swab for the pus culture and antibiotic sensitivity test, which revealed MRSA. Temporal bone CT showed the soft tissue in the middle ear encasing the ossicle (Fig. 1C). Pure tone audiogram (PTA) showed moderate to severe mixed hearing loss in the right side and severe sensorineural hearing loss in the left side (Fig. 2A).

The patient was treated for MRSA otorrhea as an outpatient with diluted vinegar irrigation (vinegar: distilled water $=1: 2$ ) and topically prepared otic solution of vancomycin. A 500-mg vial of vancomycin powder was reconstituted with $30 \mathrm{~mL}$ of sterile distilled water. The prescribed dose of vancomycin was two drops $(0.8 \mathrm{mg})$ applied topically to the ear, three times daily for a total of 14 days. After 7 days, the pulsating otorrhea continued to resolve, with the most remarkable improvement occurring by day seven of the 7-day treatment period. Three weeks after the otorrhea ceased, removal of the impression material was not achieved by transcanal approach. Afterall, the removal accomplished via a canal wall-up mastoidectomy with posterior tympanotomy approach to the middle ear (Fig. 3A, B). Silicone impression material was visible via the facial recess and was complicated by encirclement of the ossicles by the material. After removal of the granulation tissue around the ossicle, the silicone material could be removed (Fig. 3C). The ossicle was left intact. The tympanic
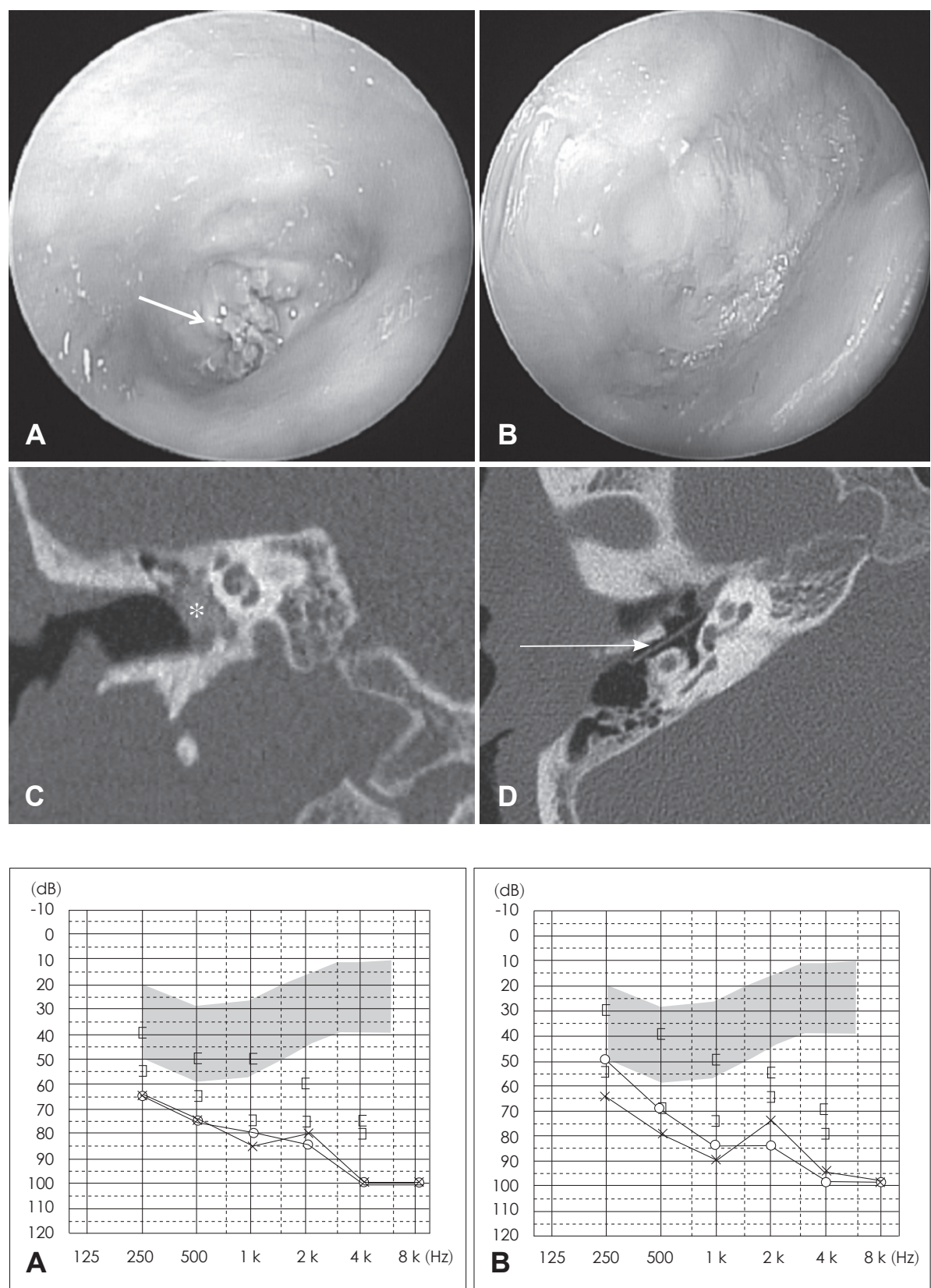

Fig. 2. A: Preoperative PTA shows that mixed type hearing loss in the right side and sensorineural hearing loss in the left side. B: The postoperative PTA shows that mild improved bone conduction in the right side. PTA: pure tone audiogram. 

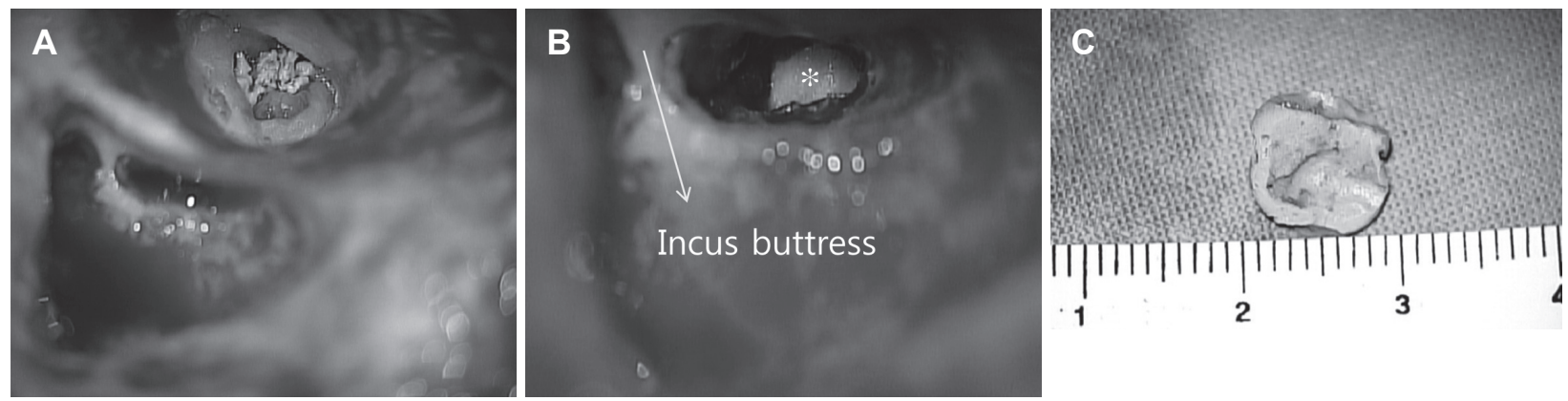

Fig. 3. A: Initially, transcanal removal was tried but the impression was fixed in the middle ear. B: The canal wall-up mastoidectomy with posterior tympanotomy revealed the silicone impression bordered by granulation tissue (asterisk indicate silicone impression; white arrow indicate position of incus buttress). C: After dissection by pick through the faciall recess, the silicone impression was removed. The removed silicone impression.

membrane perforation was reconstructed by temporalis fascia. Silastic sheet insertion from the mastoid to the middle ear via posterior tympanotomy was performed for ventilation. Vancomycin impregnated Gelfoam (Pharmacia \& Upjohn, Kalamazoo, MI, USA) was packed in the middle ear for prevention of infection. The fascia was epithelized within the first month (Fig. 1B), and the valsalva maneuver was positive. Five months later, postoperative temporal bone CT showed well ventilated middle ear and mastoid cavity (Fig. 1D). The postoperative PTA showed mildly improved bone conduction compared to preoperative PTA (Fig. 2B). The patient has worn behind-the-ear digital hearing aids in both sides.

\section{Discussion}

Fitting a hearing aid usually requires the making of an ear mold. The silicone impression material is commonly used. Although this procedure is not difficult, otoendoscopic evaluation is needed to find lesions of the external canal and the tympanic membrane perforation [5]. In the present study, the hearing aid specialist did not detect the tympanic membrane perforation before the patient underwent hearing aid fitting. Therefore, molding into the middle ear and it was not removed. To date, there have been 27 reported cases of impression material foreign bodies. However, intractable MRSA otorrhea combined with impression material foreign body has not been reported. There has been a steady increase in the number of cases of otorrhoea caused by MRSA [8]. Vancomycin is the drug of choice in treating MRSA-infected patients. Topical otic solution has an advantage because of higher levels of antibiotic in the middle ear compared to systemic injection [9]. In the present study, we treated patients with MRSA otorrhea as outpatients using a topically prepared otic solution of vancomycin with diluted vinegar irrigation.

These days, severe complications of otitis media are rare today. The availability and use of antibiotics has significantly decreased the incidence of intratemporal and intracranial complications of otitis media in south Korea. Labyrinthitis induced the most profound inner ear damage, and therefore it was the single most disabling form of intratemporal complications. Symptoms, aggravated hearing loss and vertigo were frequently encountered. The patients with labyrinthitis developed severe hearing loss or deafness. In the present study, patient did not show nystagmus and the dizziness improved immediately after administering antihistamine medication. Ahlthough his hearing was subjectively worse on the right side before undergoing hearing aid fitting. However, causal relationship was established due to lack of pre-complication audiograms.

Most reported patients had either conductive, mixed, or sensorineural hearing loss, due to the foreign body and/or extraction surgery. In this particular case, the patient had preexisting mixed hearing loss at the right side and sensorineural hearing loss at the left side. The hearing deterioration occurred due to the foreign bodies at the right side. Silicone impression was removed by transcanal sharp dissection with success [1-6]. However, this often requires disrupting the continuity of the ossicular chain to completely remove the material if this has not previously occurred secondary to erosion from the mold material $[1,2]$.

In the present case, the silicone impression material was removed by canal wall-up mastoidectomy. Through the facial recess, the ossicle was dissected from the silicone mold material. The granulation tissues were removed from the ossicle and middle ear mucosa. Although the MRSA discharge was stopped, the remaining biofilm on the granulation tissues could induce a postoperative infection and wound disruption. Therefore, we removed as much of the granulation tissue as possible. After irrigation with vancomycin diluted in saline, middle ear packing was performed using vancomycin solution impregnated gelfoam for prevention of postop- 
erative infection. The patient showed no postoperative infection and the fascia graft dried up well. Five months later, postoperative temporal bone CT showed well ventilated middle ear and mastoid cavity.

In conclusion, if impression material in the middle ear causes infection, it can induce potential serious complications. Adequate preoperative treatment for otorrhea and delayed surgical treatment is necessary. In this study, we treated MRSA otorrhea by topical vancomycin otic solution and diluted vinegar irrigation. After otorrhea ceased, we removed the impression material by canal wall-up mastoidectomy. This case showed that otoscopic examination of the tympanic membrane perforation is important. If a tympanic membrane perforation is exists, presence of impression material in the middle ear can lead to complication during the procedure of hearing aid fitting. Therefore, through otoscopic examination is essential before undergoing hearing aid fitting. Further we recommend that hearing aid fitting should be performed by an otolaryngologist instead of a hearing aid specialist.

\section{Conflicts of interest}

The authors have no financial conflicts of interest.

\section{REFERENCES}

1) Hof JR, Kremer B, Manni JJ. Mould constituents in the middle ear, a hearing-aid complication. J Laryngol Otol 2000;114:50-2.

2) Kohan D, Sorin A, Marra S, Gottlieb M, Hoffman R. Surgical management of complications after hearing aid fitting. Laryngoscope 2004; $114: 317-22$

3) Jacob A, Morris TJ, Welling DB. Leaving a lasting impression: ear mold impressions as middle ear foreign bodies. Ann Otol Rhinol Laryngol 2006;115:912-6.

4) ENT today. Management of an unusual middle ear foreign body [cited 2016 Jun 14]. Available from: http://www.enttoday.org/article/ managment-of-an-unusual-middle-ear-foreign-body/.

5) Kiskaddon RM, Sasaki CT. Middle ear foreign body. A hearing aid complication. Arch Otolaryngol 1983;109:778-9.

6) Suzuki N, Okamura K, Yano T, Moteki H, Kitoh R, Takumi Y, et al. Silicone impression material foreign body in the middle ear: two case reports and literature review. Auris Nasus Larynx 2015;42:419-23.

7) Wynne MK, Kahn JM, Abel DJ, Allen RL. External and middle ear trauma resulting from ear impressions. J Am Acad Audiol 2000; 11:351-60.

8) Jang CH, Song CH, Wang PC. Topical vancomycin for chronic suppurative otitis media with methicillin-resistant Staphylococcus aureus otorrhoea. J Laryngol Otol 2004;118:645-7.

9) Yang $\mathrm{HC}$, Cho YB, Jang CH. Efficacy of tympanoplasty without mastoidectomy on MRSA-infected chronic otitis media. Otol Neurotol 2014;35:976-80. 